2. V. Garten, Über Tauber'she Konstanten bei Cesaro'schen Mittlebildung, Comment. Math. Helv. 25 (1951), 311-335.

3. P. Hartman, Tauber's theorem and absolute constants, Amer. J. Math. 69 (1947), 599-606.

4. A. Jakimovski (Amir), Some relations between the methods of summability of Abel, Borel, Cesaro, Hölder and Hausdorff, J. Analyse Math. 3 (1953/54), 346-381.

5. - Tauberian constants for the $[J, f(x)]$ transformations, Pacific J. Math. (to appear).

The Hebrew University

\title{
WHITTAKER'S CONSTANT FOR LACUNARY ENTIRE FUNCTIONS
}

HERBERT S. WILF

1. Introduction. Let

$$
f(z)=\sum_{\nu=0}^{\infty} \frac{b_{\nu}}{\nu !} z^{\nu}
$$

be an entire function of exponential type $\tau<\infty$. We are concerned here with two problems which are closely related to the determination of Whittaker's constant, that is to say, with theorems to the effect that if $f(z)$ and each of its derivatives have some zeros in the unit circle then $\tau$ cannot be too small.

Definition 1. The constant $W_{p}$ is the largest number $W$ for which the following assertion is true: "Let the coefficients $b_{\nu}$ of $f(z)$ vanish except for values of $\nu$ in the arithmetic progression $q, q+p, q+2 p, \cdots$. If $f(z), f^{\prime}(z), \cdots$ each have a zero in $|z|<1$, and if $\tau<W$, then $f(z) \equiv 0$."

One sees, by considering $f^{(q)}(z)$ that $W_{p}$ is independent of $q . W_{1}$ is Whittaker's constant, whose value is unknown [1]. The case $p=2$ has also been investigated $[2 ; 3]$.

Definition 2. The constant $\omega_{p}$ is the largest number $\omega$ for which the following assertion is true: "Let $f(z), f^{\prime}(z), \cdots$ each have at least $p$ zeros in $|z|<1$. If $\tau<\omega$, then $f(z) \equiv 0$."

Again $\omega_{1}=W_{1}$ is Whittaker's constant. Erdös-Rényi [6, equation (15)] have shown that

$$
\omega_{p} \geqq p / e \quad(p=1,2, \cdots) .
$$

We shall give a somewhat better bound.

Presented to the Society, July 5, 1961; received by the editors February 2, 1962. 
2. The constants $W_{p}$. Our main result is

Theorem 1. The asymptotic expansions, for $p \rightarrow \infty$, of $W_{p}$ and of $(p !)^{1 / p}$, in terms of powers of $p$ and $\log p$, are identical. In particular,

$$
W_{p}=\frac{p}{e}+\frac{\log p}{2 e}+\log \sqrt{2 \pi}+o(1) \quad(p \rightarrow \infty) .
$$

To prove this, let us define

$$
h(z)=\sum_{m=1}^{\infty} \frac{z^{m p}}{(m p) !}
$$

and then state

LEMmA 1. The constant $W_{p}$ is not greater than the modulus of the root of smallest modulus of $h(z)=-1$, nor is it smaller than the unique positive real root of $h(z)=+1$.

In fact, we have

$$
\begin{aligned}
f^{(q+n p)}\left(a_{n}\right)=0 & =\sum_{\nu=0}^{\infty} \frac{b_{q+\nu+n p}}{\nu !} a_{n}^{\prime} \\
& =\sum_{m=0}^{\infty} \frac{b_{q+(n+m) p}}{(m p) !} a_{n}^{m p}
\end{aligned}
$$

for some $\left|a_{n}\right| \leqq 1(n=0,1, \cdots)$. Hence

$$
\left|b_{q+n p}\right| \leqq \sum_{m=1}^{\infty} \frac{\left|b_{q+(n+m) p}\right|}{(m p) !} \quad(n=0,1, \cdots) .
$$

Suppose $h(\tau)<1$. Choose $\epsilon$ so that $h(\tau+\epsilon)<1$. Since $f(z)$ is of type $\tau$,

$$
\left|b_{q+n p}\right| \leqq A(\tau+\epsilon)^{q+n p} \quad(A=A(\epsilon)) .
$$

Inductively, suppose it has been shown that

$$
\left|b_{q+n p}\right| \leqq A(\tau+\epsilon)^{q+n p}\{h(\tau+\epsilon)\}^{r}
$$

for some $r$. Then substitution of (6) into (5) yields (6) with $r$ replaced by $r+1$, hence (6) is true for every $r$. Since $h(\tau+\epsilon)<1, f(z) \equiv 0$ and the second half of Lemma 1 is proved. Next if $\lambda$ is the modulus of the zero of smallest modulus of $1+h(z)$ then put

$$
g(z)=1+h(\lambda z)
$$

Then $g^{(p)}(z)=\lambda^{p} g(z)$, and $g(z)$ is of type $\lambda$, whence $W_{p} \leqq \lambda$, completing the proof of the lemma.

Now consider the equation $h(x)=1$, and put $y=x^{p} / p$ !, getting 


$$
1=y+y^{2} \frac{p !^{2}}{(2 p) !}+y^{3} \frac{p !^{3}}{(3 p) !}+\cdots .
$$

Since the root we seek is surely in $0<y<2$, we have

$$
\left|y^{3} \frac{p !^{3}}{(3 p) !}+\cdots\right| \leqq y^{2} \frac{(p !)^{2}}{(2 p) !} \frac{3}{3^{p}-2}
$$

for such $y$. Putting $(p !)^{2}(2 p) !^{-1}=\delta$, we find for the positive root of (7)

$$
y=1-y^{2} \delta\left(1+O\left(3^{-p}\right)\right)
$$

and since $\delta \sim(\pi p)^{1 / 2} 2^{-2 p-1}$

$$
y=1-\delta+O\left(p \cdot 12^{-p}\right) .
$$

Hence the positive root of $h(x)=1$ is of the form ${ }^{1}$

$$
(p !)^{1 / p}\left\{1-\frac{\delta}{p}+O\left(12^{-p}\right)\right\} \quad(p \rightarrow \infty) .
$$

For the equation $h(z)=-1$ we use the fact that the function

$$
h\left(z^{1 / p}\right)+1=\sum_{m=0}^{\infty} \frac{z^{m}}{(m p) !}
$$

has only real zeros $([4], 5,160)$. Being of order $1 / p$ it has the form

$$
1+h\left(z^{1 / p}\right)=\prod_{n=1}^{\infty}\left(1+\frac{z}{\alpha_{n}}\right) \quad\left(0<\alpha_{1} \leqq \alpha_{2} \leqq \cdots\right) .
$$

We may now use the method of Euler exactly as in $[5$, p. 500] and find that

$$
\alpha_{1} \leqq\left\{\frac{p !}{1-\frac{2(p !)^{2}}{(2 p) !}}\right\}^{1 / p}
$$

Theorem 1 now follows from (8), (10) and Lemma 1.

3. The constant $\omega_{p}$. In terms of the Bessel function

$$
I_{0}(x)=\sum_{n=0}^{\infty} \frac{\left(\frac{x}{2}\right)^{2 n}}{(n !)^{2}}
$$

\footnotetext{
${ }^{1}$ I am indebted to Professor de Bruijn for this estimate. My own method gave only the first two terms in (3).
} 
we shall prove, by a similar argument,

ThEOREM 2. We have

$$
\omega_{p} \geqq \frac{1}{2} \max _{x>0}\left\{\frac{x}{\left[I_{0}(x)\right]^{1 / 2 p}}\right\} \quad(p=1,2,3, \cdots)
$$

$$
\geqq \frac{p}{e}+\frac{\log p}{4 e}+O(1) \quad(p \rightarrow \infty) .
$$

Proof. If $f^{(k)}(z)$ has $p$ zeros in $|z|<1$, then by Jensen's theorem,

(13) $p \log \rho \leqq \frac{1}{2 \pi} \int_{0}^{2 \pi} \log \left|f^{(k)}\left(\rho e^{i \theta}\right)\right| d \theta-\log \left|f^{(k)}(0)\right| \quad(\rho>1)$.

Instead of estimating the integral with the maximum modulus, we can do somewhat better with the well-known inequality of the geometric mean and r.m.s.,

$$
\exp \left\{\frac{1}{b-a} \int_{a}^{b} g(x) d x\right\} \leqq\left\{\frac{1}{b-a} \int_{a}^{b} g^{2}(x) d x\right\}^{1 / 2} .
$$

Indeed, exponentiating (13), using (14) and Parseval's identity we get

$$
\rho^{p} \leqq \frac{1}{\left|b_{k}\right|}\left\{\sum_{\nu=0}^{\infty} \frac{\left|b_{\nu+k}\right|^{2}}{(\nu !)^{2}} \rho^{2 \nu}\right\}^{1 / 2} \quad(\rho>1) .
$$

Just as in the step (5), (6), the assumption

$$
\left|b_{k}\right| \leqq A(\tau+\epsilon)^{k} \quad(k=0,1,2, \cdots)
$$

in (15) leads to

$$
\left|b_{k}\right| \leqq A(\tau+\epsilon)^{k}\left\{\frac{I_{0}(2 \rho(\tau+\epsilon))^{1 / 2}}{\rho^{p}}\right\} \quad(\rho>1) .
$$

The obvious induction shows that if the quantity in braces in (17) is less than unity, then $f(z) \equiv 0$. Hence, for all $\rho>1$

$$
I_{0}(2 \rho \tau)^{1 / 2} \geqq \rho^{p}
$$

or

$$
\tau \geqq \frac{1}{2} \max _{\rho>1} \frac{I_{0}^{-1}\left(\rho^{2 p}\right)}{\rho}=\frac{1}{2} \max _{x>0}\left\{x I_{0}(x)^{-1 / 2 p}\right\} .
$$


The asymptotic relation shown in (12) follows at once from choosing $x=2 p$ and using well-known asymptotic formulas for $I_{0}(x)$. The table below shows in the first row, $p$, in the second, the constant on the right side of (12) and in the third $p / e$.

\begin{tabular}{|c|c|c|}
\hline 1 & 2 & 3 \\
\hline 0.6897 & 1.100 & 1.493 \\
\hline 0.368 & 0.736 & 1.104 \\
\hline
\end{tabular}

\section{REFERENCES}

1. R. P. Boas, Entire functions, Academic Press, New York, 1954.

2. - Entire functions of exponential type, Bull. Amer. Math. Soc. 48 (1942), 839-849.

3. V. Ganapathy Iyer, $A$ property of the zeros of the successive derivatives of integral functions, J. Indian Math. Soc. 2 (1937), 289-294.

4. G. P6lya and G. Szegö, Aufgaben and Lehrsätze aus der Analysis, Springer, Berlin, 1954.

5. G. N. Watson, A treatise on the theory of Bessel functions, Cambridge Univ. Press, 1944.

6. P. Erdös and A. Rényi, On the number of zeros of successive derivatives of entire functions of finite order, Acta Math. 8 (1957), 223-225.

UNIVERSITY OF ILLINOIS 